activator Forskolin and the phosphodiesterase 4 (PDE4) inhibitor Roflumilast (all $1 \mu \mathrm{m}, 3 \mathrm{~d}$ ) was evaluated. IL-1 $\beta$ ( $2 \mathrm{ng} / \mathrm{ml}$, 24hr) was used for cyclooxygenase 2 (COX-2) induction. $\alpha$-smooth muscle actin ( $\alpha$-SMA, a myofibroblast marker), COX-2, EP2, EP4 and $\beta_{2}$-receptor expression was analysed by Western blotting and immunocytochemistry, respectively. Adenylyl cyclase mRNA was measured by qPCR and cAMP was measured by radioimmunoassay.

Results F-IPF showed increased $\alpha$-SMA and collagen expression and repressed COX-2 expression compared to F-NL. PGE treatment $_{2}$ prevented TGF- $\beta 1$-induced $\alpha$-SMA expression and COX- 2 repression in F-NL, which was mimicked by $\beta_{2}$-agonists and Forskolin. $\mathrm{PGE}_{2}$ also reduced $\alpha$-SMA expression and increased COX-2 expression in F-IPF despite that it induced significantly less cAMP than in F-NL But this effect on F-IPF was not mimicked by $\beta_{2}$-agonists and

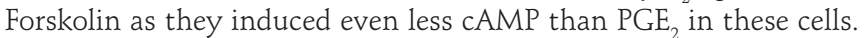
TGF- $\beta$-treated F-NL also produced less cAMP than untreated cells in response to these cAMP. stimulants. However, the expression of EP2, EP4, $\beta_{2}$-adrenoceptors and adenylyl cyclase isoforms was similar in F-NL and F-IPF. Furthermore, combination of $\mathrm{PGE}_{2}$ with Roflumilast showed greater effect than $\mathrm{PGE}_{2}$ alone on $\alpha$-SMA reduction and COX-2 expression in F-IPF and F-NL, whereas Roflumilast alone had no effect.

Conclusions $\mathrm{CAMP}$ is a key anti-fibrotic regulator of myofibroblast differentiation. However, cAMP production in myofibroblasts is defective, probably due to increased degradation by PDE4.

\section{S130 EXOGENOUS MACROPHAGES ARE RETAINED IN MOUSE LUNGS AFTER INJURY AND TARGET THERAPEUTIC TRANSGENES TO THE INJURED LUNG PARENCHYMA}

doi:10.1136/thoraxjnl-2012-202678.135

K McNulty, EK Sage, R Alexander, CJ Scotton, SM Janes. University College London, London, UK

Introduction Pulmonary fibrosis evolves in response to epithelial injury in a number of lung diseases, and carries a poor prognosis; novel therapies are urgently needed. The epithelial mitogen keratinocyte growth factor (KGF) has been shown to prevent fibrosis in a number of animal models however its therapeutic utility is limited by its short half-life. There is a growing interest in cell therapy approaches, and we hypothesised that macrophages could be used as vehicles to target KGF therapy to injured lung.

Methods Lentiviral vectors expressing luciferase, KGF and GFP (control) were generated and used to transduce the IC-21 macrophage cell line. Appropriate transgene expression was confirmed. KGF macrophages were co-cultured with primary mouse tracheal epithelial cells in a proliferation bioassay. Luciferase-expressing macrophages were tracked longitudinally using bioluminescence imaging after oropharyngeal delivery to the lungs of mice given bleomycin to induce injury, or saline control. Immunostaining was used to localise macrophages within lung sections. KGF and GFPmacrophages were delivered during bleomycin-induced lung injury; endpoint measures included lung histology, micro-CT analysis, and quantification of inflammatory cell infiltrates, vascular leak, lung collagen by HPLC, and inflammatory and fibrotic mediators by ELISA and qPCR.

Results Exogenously delivered macrophages were retained in the lungs of bleomycin-injured mice, but not uninjured controls, when given during either the inflammatory or fibrotic phases of injury, and localised to injured lung parenchyma. KGF-transduced macrophages induced proliferation of mouse tracheal epithelial cells during coculture, but delivery to bleomycin-injured mice was not associated with overall improvements in endpoints when delivered during either the inflammatory or fibrotic phases of injury. Delivery of macrophages per se was associated with an increase in inflammatory mediators consistent with classical M1 macrophage activation, which may have off-set any beneficial effects of KGF-transduced macrophages.
Conclusions Exogenously delivered macrophages are preferentially retained in injured lung and show potential as vehicles to target therapeutic transgenes by localising to damaged areas. Whilst KGF-transduced macrophages induced epithelial proliferation in vitro, any protective effects in vivo may have been negated by the exacerbatory effects of macrophage delivery. Future work will determine whether ex vivo manipulation of macrophage phenotype can confer therapeutic benefit.

\section{Occupational lung disease}

\section{S131 IDENTIFYING OCCUPATIONAL ASTHMA AMONG A COHORT OF CLEANERS IN THE NORTH EAST ENGLAND}

doi:10.1136/thoraxjnl-2012-202678.136

'S Alfajijam, ${ }^{2} \mathrm{C}$ Stenton, 'T Pless-mulloli, 'D Howel. 'Institute of Health and Society, Newcastle Upon Tyne, United Kingdom; 'NHS Foundation Trusts, Newcastle Upon Tyne, United Kingdom

Introduction We have demonstrated a prevalence of asthma of 14\% in a survey of 1400 UK hospital and university cleaners, and an estimated incidence of asthma of 3.3/1000 person-years. $26 \%$ of cleaners reported work-related symptoms. We have explored the possibility that these cleaners have occupational asthma using serial measurements peak expiratory flow (PEF) and airway responsiveness.

Objectives To identify occupational asthma in a cohort of cleaners.

Methods A respiratory symptom questionnaire was distributed among 1400 cleaners working in three local hospital trusts and two universities. Airway responsiveness (PD20) was measured in those with asthma symptoms using a methacholine challenge test. Those with measurable airway responsiveness (PD20<1600ug) were invited to undergo a repeat measurement away from work and to carry out serial PEF measurements that were analysed for a workrelated effect using OASYS (Burge, Pantin et al. 1999).

Results 557 (40\%) returned the questionnaire and 167 reported respiratory symptoms. Of these, 56 (33.5\%) attended for methacholine challenge testing. $26(46 \%)$ had quantifiable results.

12 subjects underwent serial PD20 measurements at and away from work. Overall, there were no significant changes in airway responsiveness. Geometric mean PD20 at work was 193 ug and away from work was $254 \mathrm{ug}(\mathrm{t}=0.6 ; \mathrm{p}=0.5) .5$ cleaners showed a 3 -fold or more increase in PD20 away from work raising the possibility of significant changes in those individuals.

10 subjects completed serial peak expiratory flow measurements. The mean OASYS score was 1.97. One subject had a score of $>2.5$ suggesting a work related effect.

Conclusion Although the prevalence of asthma symptoms in our cohort is consistent with other epidemiological evidence showing a 1.5 to 2.0 fold risk of asthma, we found little evidence of occupational asthma using conventional clinical diagnostic tests in this group. The findings are consistent with the hypothesis that cleaners develop their asthma in an unusual way, possibly though a low dose irritant mechanism

Burge, P.S., C.F.A. Pantin, et al. (1999). "Development of an expert system for the interpretation of serial peak expiratory flow measurements in the diagnosis of occupational asthma." Occupational and Environmental Medicine 56(11): 758-764.

\section{S132 CHRONIC BRONCHITIS, PULMONARY FUNCTION, AND OCCUPATIONAL EXPOSURE IN FRAMINGHAM HEART STUDY}

doi:10.1136/thoraxjnl-2012-202678.137

SY Liao, X Lin, DC Christiani. Harvard School of Public Health, Boston, United States 